\title{
Reconstruction of calvarial bone defects using an osteoconductive material and post-implantation hyperbaric oxygen treatment
}

\author{
Tim Mo Chen ${ }^{\mathrm{a}, *}$, Chung Shih ${ }^{\mathrm{b}}$, Teng Fu Lin ${ }^{\mathrm{c}}$, Feng Huei $\operatorname{Lin}^{\mathrm{d}}$ \\ ${ }^{a}$ Division of plastic surgery, Department of surgery, Tri-Service General Hospital, National Defense Medical Center, Taipei, Taiwan \\ ${ }^{\mathrm{b}}$ Department of Anatomy and Biology, National Defense Medical Center, Taipei, Taiwan \\ ${ }^{\mathrm{c}}$ Department of Undersea and Hyperbaric Medicine, Tri-Service General Hospital, National Defense Medical Center, Taipei, Taiwan \\ ${ }^{\mathrm{d}}$ Center for Biomedical Engineering, College of Medicine, National Taiwan University, Taipei, Taiwan
}

Received 10 June 2004; received in revised form 30 June 2004; accepted 11 August 2004

Available online 2 October 2004

\begin{abstract}
We have developed a biodegradable, malleable, osteoconductive material for calvarial bone defect reconstruction, and evaluated the extent of new bone formation in rabbits after its implantation followed by hyperbaric oxygen (HBO) treatment. Critical-sized calvarial bone defects ( $15 \mathrm{~mm}$ in diameter) were created in 40 New Zealand white rabbits, and reconstructed using the osteoconductive material. Twenty rabbits were given 30 sessions of intermittent HBO treatment (pure oxygen at 2.4 atmospheric absolute pressure (ATA) for 90 min) after surgery; the others, without HBO treatment, served as controls. Sequential fluorescent labeling was performed after surgery. The animals were euthanized 3 months after surgery and bone specimens were subjected to microradiographic, histological, and histomorphometric evaluations. The results demonstrate that there was significantly more new bone formation in the HBO group compared with the controls (microradiogram: $85.2 \pm 4.2 \%$ vs. $32.5 \pm 2.2 \%$ of the original bone defect, histomorphometry: $70.2 \pm 3.4 \%$ vs. $30.8 \pm 2.4 \%$ of the original bone defect, $p<0.05$ ), and sequential florescent labeling demonstrated coalescence of the active mineralization elements in the HBO group. The use of this novel material with HBO treatment may offer an alternative to autogenous bone grafting or methyl methacrylate for calvarial bone defect reconstruction.
\end{abstract}

(C) 2004 Elsevier B.V. All rights reserved.

Keywords: Osteoconduction; Hyperbaric oxygen; Calvarial bone defect

\section{Introduction}

Reconstruction of the post-traumatic, full-thickness, calvarial bone defect is needed to provide brain protection, to restore aesthetic contours, and to correct intracranial ventricular collapse.

Currently the two most widely used materials for calvarial bone defect reconstruction are autogenous bone graft and methyl methacrylate. Both of them are less than ideal. Autogenous bone grafts can undergo unpredictable resorption, are of limited quantity, and may result in donor site morbidity [1-4]. Methyl methacrylate can cause a

\footnotetext{
* Corresponding author. Tel.: +886 2 87927195; fax: +886 2 87927194

E-mail address: timmo@ms22.hinet.net (T.M. Chen).
}

marked inflammatory response and fibrous encapsulation of the implant, resulting in the possibility of infection, loosening, and exposure of the implant [5].

In the search for alternatives to autogenous bone graft and methyl methacrylate, much effort has been directed to the development of osteoconductive materials composed of various calcium phosphate compounds because of closed chemical and crystal resemblance of these materials to natural bone mineral. The osteoconductive material would aid in the repair of the bone defect by acting, preferably temporarily, as a scaffold for capillaries and osteoprogenitor cells ingrowth [6]. The most widely investigated osteoconductive materials are tricalcium phosphate $\mathrm{Ca}_{3}\left(\mathrm{PO}_{4}\right)_{2}$ and hydroxyapatite $\mathrm{Ca}_{10}\left(\mathrm{PO}_{4}\right)_{6}(\mathrm{OH})_{2}$ [7]. When shaped in porous block, calcium phosphate ceramics are brittle, nonmalleable and difficult to fit the calvarial bone defect $[8,9]$. 
Granular or cement form of calcium phosphate can be packed to fit the bone defect, but they tend to absorb the ambient fluid and are difficult to contain within the area requiring reconstruction [10-20]. In addition, as a result of the microporous nature of the calcium phosphate cement, minimal bony or vascular ingrowth occurs [21].

To improve the mechanical properties of calcium phosphate compounds, we have developed a biodegradable, malleable, osteoconductive material consisting of tricalcium phosphate particles bound by gelatin which is set by glutaraldehyde mediated cross-linking. Gelatin, the partially hydrolyzed collagen extracted and purified from porcine skin, not only can hold the tricalcium phosphate particles in place, but can also increase the mechanical strength of the material so that it can be malleable without resultant fracture.

The gelatin matrix degrades in vivo with time, allowing new bone ingrowth around the tricalcacium phosphate particles. The biological effect of this material has been studied by co-culture of the material with rat osteoblasts [22]. It was found that calcium ions and gelatin were gradually released from the composite material and served as nutrients for the growth of osteoblasts. The results also demonstrated good adhesion and spreading of the osteoblasts on the surface of the material (Fig. 1). The biocompatibility and osteoconductivity of the material has also been studied with a critical-sized calvarial bone defect model in rabbits [23], and the results demonstrated that the material was biodegradable with progressive replacement by new bone in a centripetal direction.

Since tricalcium phosphate compounds lack of osteoindcutive potential and due to the paucity of bone marrow interposed between the diploe of the calvarium, an agent is needed to accelerate the osteoconductivity of this material so that more new bone can be generated. Hyperbaric oxygen (HBO) is a currently available modality to accelerate the

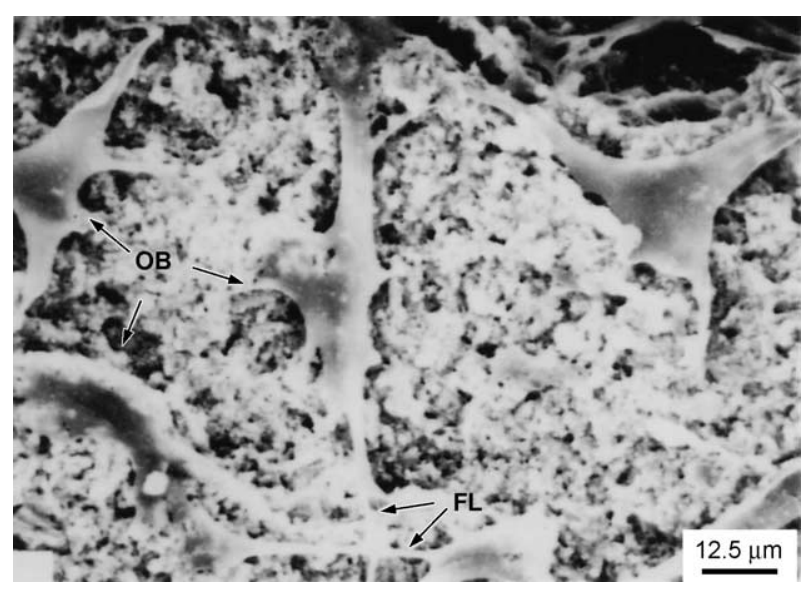

Fig. 1. Scanning electron micrograph of rat osteoblasts (OB) attached to the surface of the osteoconductive material showing flattened morphology and filament (FL) formation indicating cytoplasmic ruffling. (Reprinted with permission from Ref. [22]). activity of osteogenesis in clinical practice. The use of HBO as an adjunct for bony reconstruction of the mandible in oral cancer patients has been well documented [24]. To the best of our knowledge, the role of HBO in promoting osteogenesis after implantation of an osteoconductive material has not been fully elucidated, The purpose of this study is to evaluate the extent of new bone formation subsequent to implantation of such material with post-implantation HBO treatment for critical-sized calvarial bone defects in rabbits.

\section{Materials and methods}

\subsection{Osteoconductive material preparation}

Tricalcium phosphate powder (Merck, Darmstadt, Germany) was placed in a platinum crucible, sintered at $1000{ }^{\circ} \mathrm{C}$ for $1 \mathrm{~h}$, and then cooled to room temperature. The sintered powder was crushed in a grinding bowl and sieved through 40-60 mesh. Particles with an average grain size of 100-150 $\mu \mathrm{m}$ were thus obtained for material preparation. The sintered tricalcium phosphate is pure beta phase as determined by Xray diffraction spectra. Following this, $5 \mathrm{~g}$ of gelatin powder (Sigma, St Louis, MO, USA) was dissolved in $25 \mathrm{ml}$ of deionized distilled water at $65{ }^{\circ} \mathrm{C}$ using a water bath. This homogeouns gelatin solution was mixed with $15 \mathrm{~g}$ tricalcium phosphate particles. Glutaraldehyde solution (4\%, Sigma) was then added to the tricalcium phosphate/gelatin matrix for gelatin matrix cross-linking. The final concentration of glutaraldehyde in the osteoconductive material was $0.05 \%$ $\mathrm{v} / \mathrm{v}$. The material was manually packed into $15 \mathrm{~mm}$ in diameter, $2.0 \mathrm{~mm}$ in thickness, cylindrical Teflon molds and dried overnight in an oven to produce pre-formed material. The material was sterilized by autoclaving before use.

\subsection{Animals}

Forty mature New Zealand white rabbits, weighing 3.0 $3.5 \mathrm{~kg}$, were used. The study was approved by the Scientific Committee of the National Defense Medical Center, Taiwan, and was carried out according to the regulations of the National Institutes of Health of Taiwan.

\subsection{Surgical procedures}

The rabbits were anesthetized with intramuscular injections of sodium pentobarbital $(40 \mathrm{mg} / \mathrm{kg})$. The head was shaved and disinfected with Betadine. The parietal bone was exposed by a midline incision and the overlying periosteum was incised. A 15-mm-diameter, full-thickness bone defect was created using a drilling burr in a slow-speed dental handpiece supplemented with $0.9 \%$ sterile saline irrigation. The dura and superior sagittal sinus were not violated. A bone defect of this size in a rabbit will not spontaneously heal during a life time and is thus defined as a critical-sized defect [25]. The bone defect was then reconstructed with the 


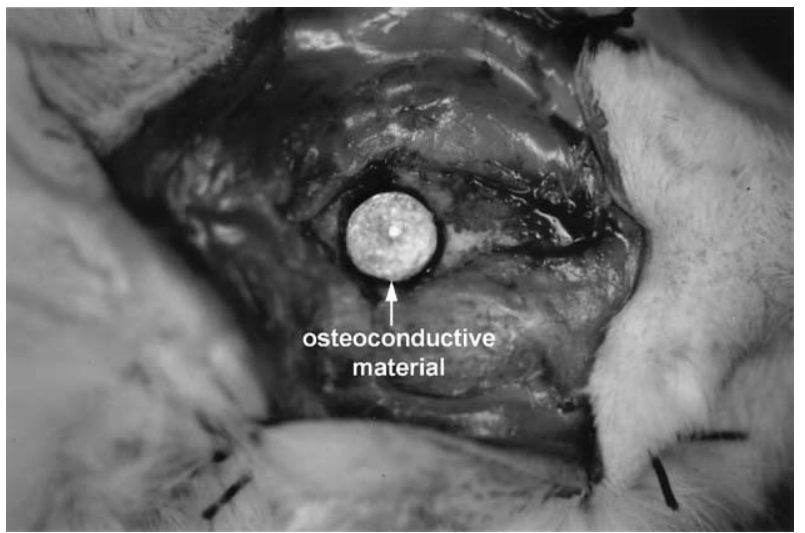

Fig. 2. Reconstruction of a 15-mm-diameter, full-thickness, calvarial bone defect using a malleable, biodegradable, osteoconductive material.

osteoconductive material. The material was easily molded to fit the bone defect and did not require fixation (Fig. 2). The periosteum was closed with 5-0 vicryl sutures and the skin was closed with 4-O nylon. Cefazolin (100 mg/kg) was administered preoperatively and $12 \mathrm{~h}$ after surgery. Analgesic (Metamizole sodium $50 \mathrm{mg} / \mathrm{kg}$ ) was administered in the immediate post-operative period. The rabbits were in single cages under standard environmental conditions. They were maintained on commercially available rodent food and water ad libitum. The rabbits could move about freely.

\subsection{Hyperbaric oxygen treatment}

An experimental hyperbaric chamber was used for the study. The chamber was pressurized with pure oxygen. All $\mathrm{CO}_{2}$ produced by the animals was eliminated by a constant flow of $\mathrm{O}_{2}$. Twenty rabbits were exposed daily to hyperbaric oxygen for $90 \mathrm{~min}$ at 2.4 atmospheric absolute pressure (ATA) of pure oxygen for 30 consecutive days after surgery. The other 20 rabbits were placed in the atmospheric chamber with plain air, serving as a control group.

\subsection{Specimen preparation}

Three months following surgery, the rabbits were euthanized using an overdose of sodium pentobarbital. Sequential fluorescent labeling was performed to demonstrate the mineralization front of the new bone by giving two intra-peritoneal injections of fluorescein-labeled methyleneiminodiacetic acid (15 mg/kg Calcein; Sigma), 10 and 3 days before death. Craniectomy sites with $2-3 \mathrm{~mm}$ of contiguous bone were then removed for microradiography, histology, and histomorphometry.

\subsection{Microradiography}

Microradiograms were taken of the specimens in a cabinet X-ray machine (Ohmic model OM-603, Tokyo, Japan) using Kodak high-resolution film, at $18 \mathrm{kV}$ and $3 \mathrm{~mA}$ for $3 \mathrm{~min}$. Each roentgenogram was evaluated for the area of radiopacity in the calvarial bone defect using an image analysis system with Photoshop software (version 5.0J; Adobe, Mountain View, CA, USA). The results were recorded as the percentage of the original calvarial bone defect occupied by radiopacity.

\subsection{Histology and histomorphometry}

The specimens were fixed in $70 \%$ ethanol. After dehydration, the specimens were embedded in methyl methacrylate, cut using a bone-sectioning machine (Malto Tokyo, Japan) to approximately $200 \mu \mathrm{m}$ thickness, and then ground to $10-\mu \mathrm{m}$ thickness. The non-decalcified sections were stained with Goldner-Masson trichrome. Light microscopic examinations, including histomorphometric calculations, were performed using Leitz Microvid equipment (Leitz, Wetzlar, Germany) connected to an IBM computer. The histomorphometric results were recorded as the percentage of original calvarial bone defect occupied by new bone and osteoids. Fluorescence microscopic examination was also performed to detect the mineralization front of the new bone stained by Calcein.

\subsection{Statistical analysis}

The microradiographic and histomorphometric measurements data were first tested for normality of distribution and are presented as means \pm standard deviations (SD). Student's $t$-tests were used to examine any differences between the experimental and control groups, and significance was assumed at $P<0.05$.

\section{Results}

All animals survived for the entire duration of the experiment. There was no wound infection and none of the implants became extruded. On gross inspection, the

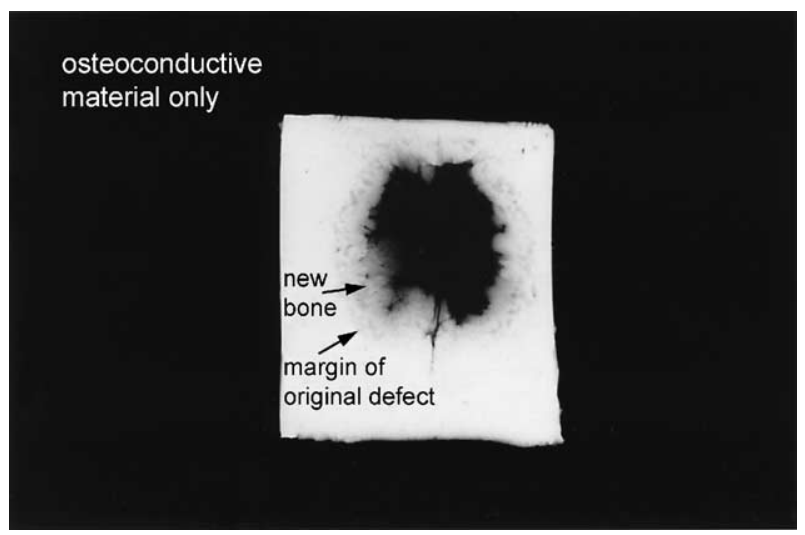

Fig. 3. Microradiography of a calvarial bone defect, 3 months after osteoconductive material implantation in the absence of HBO. New bone was laid down in a centripetal fashion and tended to obscure the original margin of the bone defect. 


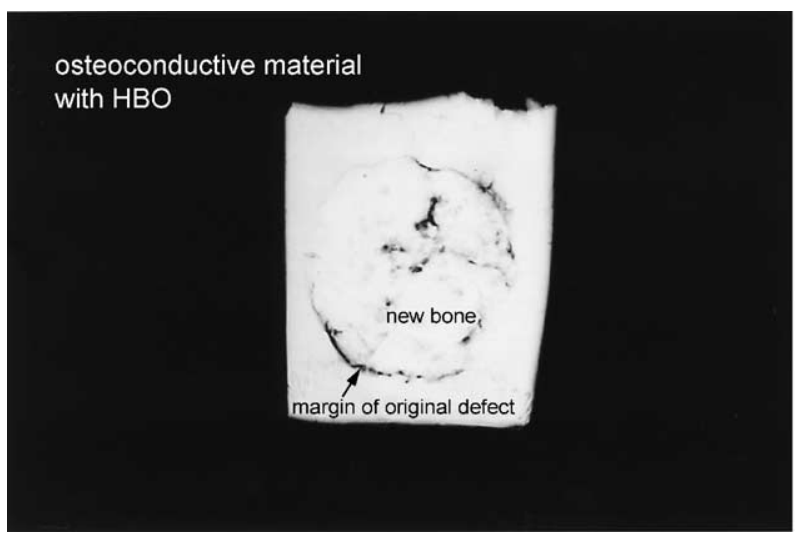

Fig. 4. Microradiography of a calvarial bone defect, 3 months after osteoconductive material implantation in the presence of HBO. Note the significantly greater extent of new bone formation occupying the defect compared with the control.

osteoconductive materials were intimately incorporated with the surrounding host bone.

\subsection{Microradiography}

Radiopaque material was laid down from the periphery of the calvarial bone defect in a centripetal fashion in the control group, and tended to obscure the original margin of the defect (Fig. 3). The mean area of radiopacity was $32.5 \pm 2.2 \%$ of the original calvarial bone defect. There was significantly more radiopaque material present in the HBO-treated group, (Fig. 4 ), with a mean area of radiopacity of $85.2 \pm 4.2 \%$ of the original calvarial bone defect ( $P<0.05$ vs. the controls).

\subsection{Histology and histomorphometry}

Sections of the sites from control animals revealed lamellar bone and osteoid tissue formation at the periphery of the calvarial bone defect (Fig. 5). Histomorphometric analysis showed that the amount of lamellar bone and

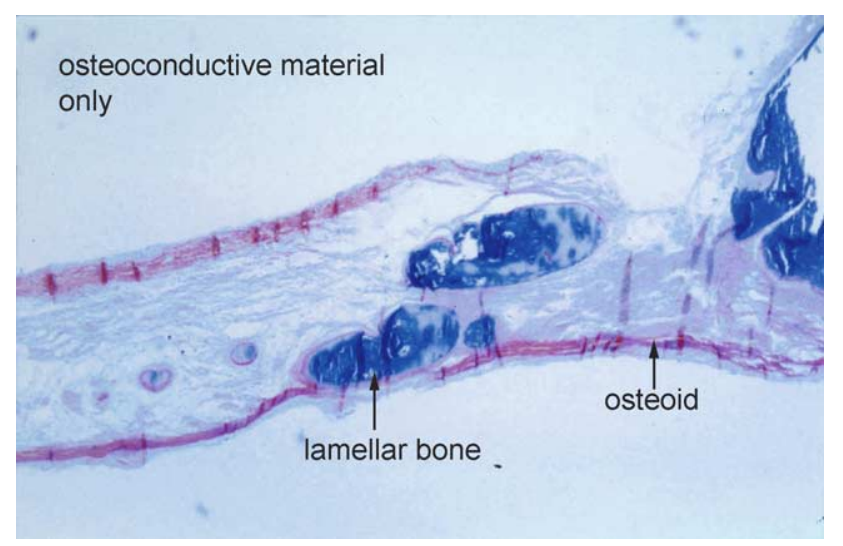

Fig. 5. Goldner-Masson trichrome-stained non-decalcified histological section of the caraniectomy site, 3 months after osteoconductive material implantation without HBO. Generation of lamellar bone and osteoid tissue can be noted at the margin of the calvarial bone defect (original magnification $\times 10$ ).

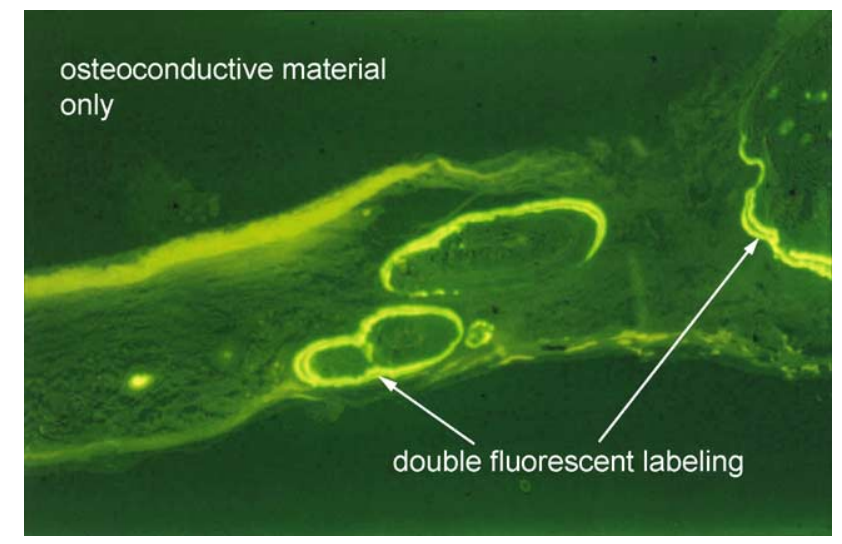

Fig. 6. Fluorescent staining of a non-decalcified histological section of the craniectomy site, 3 months after osteoconductive material implantation without HBO. Double-fluorescent labeling of the lamellar bone and osteoid tissues indicates sites of active mineralization (original magnification $\times 10$ ).

osteoid tissue was $30.8 \pm 2.4 \%$ of the original defect. Fluorescent staining revealed double-fluorescent labeling of the lamellar bone and osteoid tissue, indicating sites of active mineralization (Fig. 6).

Sections from the HBO-treated group revealed a significantly greater quantity of lamellar bone and osteoid tissue than controls: $70.2 \pm 3.4 \%$ of the original calvarial bone defect $(P<0.05$; Fig. 7). Fluorescent staining showed greater coalescence of the active mineralization elements within the new bone compared with controls (Fig. 8).

\section{Discussion}

The currently available osteoconductive materials composed of calcium phosphate for the purpose of calvarial bone defect reconstruction have limited effectiveness because of their mechanical properties. Ceramic blocks, or granular or cement forms, are far from ideal. We have developed an osteoconductive material consisting of trical-

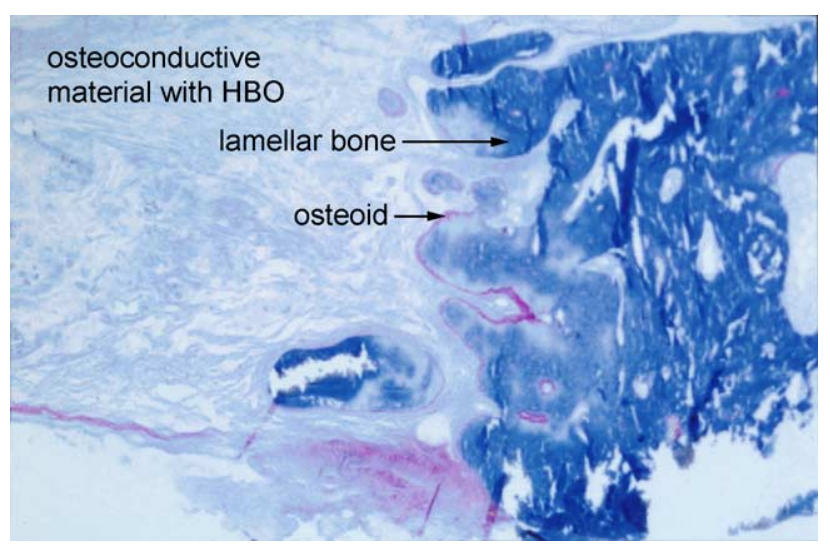

Fig. 7. Goldner-Masson trichrome-stained non-decalcified histological section of the craniectomy site, 3 months after osteoconductive material implantation in the presence of HBO. A significantly greater degree of lamellar bone and osteoid tissue formation can be noted compared with the control in Fig. 5 (original magnification $\times 10$ ). 


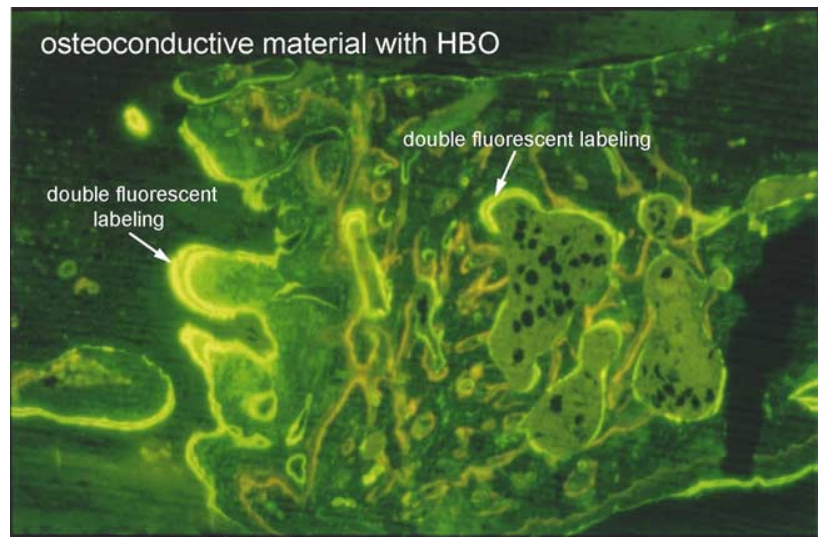

Fig. 8. Calcein-stained non-decalcified histological section of the craniectomy site, 3 months after osteoconductive material implantation in the presence of HBO. More coalescence of the active mineralization elements can be seen clearly by fluorescent staining compared with the control in Fig. 6 (original magnification $\times 10$ ).

cium phosphate particles bound by a gelatin matrix fixed by glutaraldehyde-mediated cross-linking. Our previous cell culture study in vitro and animal study with critical-sized, calvarial bone defect in rabbits have demonstrated that this material fulfils most of the criteria for an ideal osteoconductive biomaterial.

Since tricalcium phosphate compounds lack osteoinductive potential and, because of the relative lack of bone marrow in the calvarium, an agent is needed to accelerate bone growth in and around repair sites so that this material can be more useful in clinical practice. Urist et al. [26] added bone morphogenetic protein (BMP) to a tricalcium phosphate compound and implanted the mixture into the quadriceps muscle of rats. He reported 12 times as much new bone formation compared with using BMP alone. Unfortunately, the isolation and purification of BMP are both time-consuming and expensive, and its application for calvarial bone defect reconstruction is still limited, as found in preliminary clinical trials $[27,28]$.

Hyperbaric oxygen (HBO) is currently used to accelerate osteogenesis and its use as an adjunct treatment for mandible reconstruction in oral cancer patients is well documented [24]. Bone regeneration is complex and many factors have been considered in explaining the mechanism of acceleration of bone regeneration by $\mathrm{HBO}$ treatment. The factors include cellular differentiation to osseous tissue, increased collagen synthesis and angiogensis. Using tissue-culture models with multipotent mesenchymal cells, Shaw and Baset [29] showed that an increased oxygen tension elicits cellular differentiation to osseous tissue, whereas decreased oxygen tension results in cartilage formation. HBO treatment also increases the amount and rate of collagen synthesis. Collagen synthesis is oxygen dependant in two ways. First, several molecules of ATP are required to insert a single amino acid into the collagen and this energy requirement cannot be provided by anaerobic glycolysis alone. Second, oxygen is the only known substrate for hydroxylation of the key collagen amino acids proline and lysine. The collagen produced acts as a framework for endothelial proliferation from the adjacent capillaries [30]. Furthermore, angiogenesis is increased by $\mathrm{HBO}$, and plays an important role in the viability of the newly formed bone [31]. HBO treatment is intermittent, during a HBO session, the dissolved oxygen in blood becomes near 20 times as much as that at the normal status. However, $\mathrm{PaO}_{2}$ returns to preexisting levels within $10 \mathrm{~min}$ after treatment with lactate buildup in the wound bed between treatments. Tissue macrophages migrate to the wound bed and secret a variety of substances, including a chemostactic angiogensis factor, in response to the high lactate levels. Therefore, angiogenesis is increased in the wound bed by HBO treatment. Neovascularization supplies more oxygen to the central area of the wound and promotes osteogenesis. High lactate level in the wound bed is also an important stimulus to the beginning of collagen synthesis. Sawai et al. [32] demonstrated that $\mathrm{HBO}$ accelerated the union of free autogenous cortico-cancellous bone grafts derived from the iliac crest and implanted into the mandible of rabbits. Animal studies also have demonstrated that osteointegration of dental implants in irradiated bone is improved by the administration of HBO [33-36].

In this study, we demonstrated that 30 sessions of $\mathrm{HBO}$ treatment subsequent to the implantation of a malleable, biodegradable, osteoconductive material in a critical-sized calvarial bone defect of rabbits was able to significantly accelerate the extent of injury site osteogenesis compared with controls. We believe that the results of this study are significant. Clinically, such material could be implanted in areas with a low blood supply, including previously scarred or irradiated tissue. The use of post-implantation $\mathrm{HBO}$ treatment not only accelerates osteogenesis but also elicits an increase of collagen synthesis and angiogenesis. These factors are all essential for the formation and viability of solid new bone.

In summary, we have evaluated the extent of new bone formation in critical-sized calvarial bone defect model of rabbits after implantation of a biodegradable, malleable, osteoconductive material with post-implantation hyperbaric oxygen treatment. Judicious use of this combination thus offers an alternative to autogenous bone grafting or methyl methacrylate implants for the reconstruction of full-thickness, calvarial bone defects.

\section{References}

[1] W.B. Nickell, M.J. Jurkiewicz, K.E. Salyer, Repair of skull defects with autogenous bone, Arch. Surg. 105 (1972) 431.

[2] D.J. Prolo, K.P. Burres, W.T. Mclaughlin, Autogenous skull cranioplasty: fresh and preserved (frozen) with consideration of the cellular response, Neurosurgery 4 (1979) 18.

[3] S.A. Wolf, Autogenous bone grafts versus alloplastic material in maxillofacial surgery, Clin. Plast. Surg. 9 (1982) 539. 
[4] M.S.B. Edwards, D.K. Ousterhout, Autogenic skull bone grafts to reconstruct large or complex skull defects in children and adolescents, Neurosurgery 20 (1987) 273.

[5] P.N. Manson, W.A. Crawley, J.E. Hoopes, Frontal cranioplasty: risk factors and choice of cranial vault reconstructive material, Plast. Reconstr. Surg. 77 (1986) 888.

[6] C.N. Cornell, J.M. Lane, Current understanding of osteoconduction in bone regeneration, Clin. Orthop. Relat. Res. 355S (1998) S267.

[7] R.W. Bucholz, A. Carlton, R.E. Holmes, Hydroxyapatite and tricalcium phosphate bone graft substitute, Orthop. Clin. North Am. 18 (1987) 323.

[8] R.E. Holmes, H.K. Hagler, Porous hydroxyapatite as a bone graft substitute in cranial reconstruction: a histometric study, Plast. Reconstr. Surg. 81 (1988) 662.

[9] K.E. Sayler, C.D. Hall, Porous hydroxyapatite as an onlay bone graft substitute for maxillofacial surgery, Plast. Reconstr. Surg. 84 (1989) 236.

[10] M.F. Zide, J.N. Kent, L. Machado, Hydroxyapatite cranioplasty directly over dura, J. Oral Maxillofac. Surg. 45 (1987) 481.

[11] C.E. Rawling III, R.H. Winkins, J.S. Hanker, et al., Evaluation in cats of a new material for cranioplasty: a composite of plaster of paris and hydroxyapatite, J. Neurosurg. 69 (1988) 269.

[12] N.G. Georgiade, J. Hanker, G. Ruff, The use of particulate hydroxyapatite and plaster of paris in aesthetic and reconstructive surgery, Aesthet. Plast. Surg. 17 (1993) 85.

[13] H.S. Byrd, P.C. Hobar, K. Shewmake, Augmentation of the craniofacial skeleton with porous hydroxyapatite granules, Plast. Reconstr. Surg. 91 (1993) 15.

[14] F.D. Burstein, S.R. Cohen, W. Hudgins, et al., The use of porous granular hydroxyapatite in secondary orbitocranial reconstruction, Plast. Reconstr. Surg. 100 (1997) 869.

[15] P.D. Costantino, C.D. Friedman, K. Jones, et al., Experimental hydroxyapatite cement cranioplasty, Plast. Reconsr. Surg. 90 (1992) 174.

[16] J.F. Kveton, C.D. Friedman, J.M. Piepmeier, et al., Reconstruction of suboccipital craniectomy defects with hydroxyapatite cement, Laryngoscope 105 (1995) 156.

[17] A. Gosain, Hydroxyapatite cement paste cranioplasty for the treatment of temporal hollowing after cranial vault remodeling in a growing child, J. Craniofac. Surg. 8 (1997) 506.

[18] F.D. Burstein, S.R. Cohen, R. Hudgins, et al., The use of hydroxyapatite cement in secondary craniofacial reconstruction, Plast. Reconstr. Surg. 104 (1999) 1270.

[19] I.T. Jackson, R. Yavuzer, Hydroxyapatite cement: an alternative for cranofacial skeleton contour refinement, Br. J. Plast. Surg. 53 (2000) 24.

[20] C.D. Friedman, P.D. Costantino, C.H. Snyderman, et al., Reconstruction of the frontal sinus and frontofacial skeleton with hydroxyapatite cement, Arch. Facial Plast. Surg. 2 (2000) 124.

[21] D. Matic, J.H. Phillips, A contraindication for the use of hydroxyapatite cement in the pediatric population, Plast. Reconstr. Surg. 110 (2002) 1.
[22] F.H. Lin, C.H. Yao, J.S. Sun, H.C. Liu, C.W. Huang, Biological effects and cytotoxicity of the composite composed by tricalcium phosphate and glutaraldehyde cross-linked gelatin, Biomaterial 19 (1998) 905.

[23] T.M. Chen, C.H. Yao, H.J. Wang, G.H. Chou, T.W. Lee, F.H. Lin, Evaluation of a novel malleable, biodegradable osteoconductive composite in a rabbit cranial defect model, Mater. Chem. Phys. 55 (1998) 44.

[24] R.E. Marex, J.R. Ames, The use of hyperbaric oxygen therapy in bony reconstruction of the irradiated and tissue-deficient patient, J. Oral Maxillofac. Surg. 40 (1982) 412.

[25] J.P. Schmitz, J.O. Hollinger, The critical size defect as an experimental model for craniomandibulofacial nonunions, Clin. Orthop. Relat. Res. 20 (1975) 365.

[26] M.R. Urist, A. Lietze, E. Dawson, Beta-tricalcium phosphate delivery system for bone morphogenetic protein, Clin. Orthop. Relat. Res. 187 (1984) 277.

[27] H.F. Sailer, E. Kolb, Application of purified bone morphogenetic protein (BMP) preparation in cranio-maxillo-facial surgery. BMP in compromised surgical reconstructions using titanium implants, J. Craniomaxillofac. Surg. 22 (1994) 2.

[28] H.F. Sailer, E. Kolb, Application of purified bone morphogenetic protein (BMP) preparation in cranio-maxillo-facial surgery. Reconstruction in craniofacial malformations and post-traumatic or operative defects of the skull with lyophilized cartilage and BMP, J. Craniomaxillofac. Surg. 22 (1994) 191.

[29] J.L. Shaw, C.A.L. Basset, The effect of varying oxygen concentrations on osteogenesis and embryonic cartilage in vitro, J. Bone Jt. Surg. Am. 49 (1967) 73.

[30] T.K. Hunt, M.P. Pai, The effect of varying ambient oxygen tensions on wound metabolism and collagen synthesis, Surg. Gynecol. Obstet. 135 (1972) 561.

[31] R.P. Johnson, R.E. Marex, S.B. Buckley, Hyperbaric oxygen in oral maxillo-facial surgery, in controversies in oral and maxillofacial surgery, Saunders, Philadelphia, PA, 1994, p. 107.

[32] T. Sawai, A. Nimi, H. Takahashi, M. Ueda, Histologic study of the effect of hyperbaric oxygen therapy on autogenous free bone graft, J. Oral Maxillofac. Surg. 54 (1996) 975.

[33] T. Sawai, A. Nimmi, C.B. Johansson, et al., The effect of hyperbaric oxygen treatment on bone tissue reation to c.p. titanium implants placed in free autogenous bone graft, Clin. Oral Implants Res. 9 (1998) 384.

[34] P.E. Larsen, M.J. Stronczek, F.M. Beck, et al., Osteointegration of implants in radiated bone with and without adjunctive hyperbaric oxygen, J. Oral Maxillofac. Surg. 51 (1993) 280.

[35] X. Chen, Y. Matsui, K.I. Michi, K. Ohno, et al., Histomorphometric evaluation of the effect of hyperbaric oxygen treatment on healing around hydroxyapatite implants in irradiated rat bone, Int. J. Oral Maxillofac. Implants 14 (1999) 61.

[36] Å.A. Johnsson, T. Sawai, M. Jacobsson, et al., A histomorphometric study of bone reactions to titanium implants in irradiated bone and the effect of hyperbaric oxygen treatment, Int. J. Oral Maxillofac. Implants 14 (1999) 699. 